\title{
Interaction of ethanol and congener contents in aversion conditioning to alcoholic beverages in rats
}

\author{
JOSEPH J. FRANCHINA \\ Virginia Polytechnic Institute and State University, Blacksburg, Virginia
}

\begin{abstract}
One hundred rats (Rattus norvegicus) received aversion training (beverage- $\mathrm{LiCl}$ pairings) with wine and distilled spirits conditioned stimuli (CSs) whose contents were high or low in congeners. Following training, each CS group received 8 days access to a 5\% ethanol solution or to distilled water and then were tested for aversion to the original beverage CS. Groups that had received distilled water access between training and testing showed more persistent aversion effects to the wine CSs as a class than to the distilled spirits CSs. For each beverage class, aversion was greater to the CS with the higher content of congeners. Compared with distilled water access groups, groups that had received the $5 \%$ ethanol solution between training and testing showed reduced aversion effects to the beverage high in congener contents in each beverage class and no change in aversion to the low-congener beverage.
\end{abstract}

A conditioning analysis of chemical aversion therapy (CAT) for alcoholism suggests that an alcoholic beverage (e.g., wine) might be viewed as a compound stimulus of ethyl alcohol, water, and other components, such as fusel oil, esters, acetaldehyde, methanol, and tannins, which are collectively called congeners (Greizerstein, 1981; cf. Leake \& Silverman's, 1971, use of the term congeners). Since ethyl alcohol (ethanol) is in all alcoholic beverages, it seems important to know whether or not ethanol provides a functional stimulus for conditioning in the beverage compound conditioned stimulus (CS). If the ethanol cue is functional, the presence of ethanol may influence differences in conditioning between beverages of different ethanol concentrations (e.g., beer vs. Scotch). The commonality of ethanol across alcoholic beverages may also underlie the generalization of conditioning effects from one beverage to another.

Revusky and Taukulis (1975) reported reliable aversion conditioning effects to a $10 \%$ ethanol solution. Franchina, Dyer, Gilley, Ness, and Dodd (1985) suggested, however, that when ethanol is part of a beverage compound CS (e.g., whiskey), the ethanol cue may be subsidiary to cues from other beverage components. Franchina and Dyer (1986) reported that when ethanol concentration was held constant, the magnitude of aversion conditioning reliably depended on the congener content of the classes of alcoholic beverages (i.e., wine, beer, and distilled spirits).

Greizerstein (1981) reported the contents of congeners, such as acetaldehyde, ethyl formate, ethyl acetate,

The data presented in this paper were reported at the meeting of the Southeastern Psychological Association, Orlando, FL, 1986. The author thanks A. B. Dyer for critically reading an earlier draft of this paper. The author's mailing address is: Department of Psychology, Virginia Polytechnic Institute and State University, Blacksburg, VA 24061. methanol, and isopentanol, in 6 brands of beer and wine and 11 brands of distilled spirits. When the congeners are calculated per standard drink, the total congener contents of $4 \mathrm{oz}$ of wine and $12 \mathrm{oz}$ of beer were greater than those of $1.5 \mathrm{oz}$ of distilled spirits. Accordingly, if the congener contents of an alcoholic beverage contribute to a beverage's taste and aroma characteristics (Snell, 1958) and perhaps influence the beverage's conditionability (Franchina \& Dyer, 1986), then the role of the ethanol cue in conditioning may depend upon the congener level of the beverage CS.

So that I could evaluate this possibility, rats received aversion conditioning with wine or distilled-spirits CSs whose contents of congeners were high or low. After conditioning, each CS group received daily access to ethanol or to distilled water and then was tested for aversion effects to the original beverage CS. If the ethanol stimulus acquired aversive properties during conditioning with the beverage CS, repeated access to ethanol after conditioning should extinguish that aversion, thereby reducing the overall aversion to the beverage. Furthermore, if the congener level of the beverage CS mitigates the rat's conditioning to ethanol, the effects of ethanol access after conditioning may depend upon the beverage's content of congeners.

\section{METHOD}

\section{Subjects}

The rats were 100 experimentally naive male albinos (Rattus norvegicus) that were 68-80 days old. The source of animals, their housing, and the experimental room conditions were the same as those reported by Franchina et al. (1985).

\section{Design and Procedures}

Procedures for acclimating rats to water deprivation, aversion training, recovery, and testing were reported by Franchina et al. (1985). After 4 days of acclimation to water deprivation, each rat was weighed (mean 
weight $=310 \mathrm{~g} ; S E M=3.6$ ). Rats were matched by weight in groups of 8 , and then they were randomly assigned to cells of a $2 \times 2 \times 2 \times 2$ design. There were two beverage class CSs (wine or distilled spirits), two congener levels (high or low) of each class of CS, two aversion training procedures (CS paired with an injection of lithium chloride or saline), and two access fluids (ethanol or distilled water) available to the rats between training and aversion testing.

For aversion training (Days 5 and 6), all rats received $2 \mathrm{~min}$ of fluid access, followed within $5 \mathrm{~min}$ by an intraperitoneal injection at $2 \%$ body weight. On Day 5, each rat received the appropriate alcoholic beverage CS. Each rat in the conditioning (COND) groups $(n=14$ each) then received an injection of $.15 M$ lithium chloride $(\mathrm{LiCl})$, and each rat in the control groups $(n=11$ each) received an injection of $0.9 \%$ isotonic saline. On Day 6, all rats received distilled water. Rats in the COND groups received saline injections; rats in the control groups received injections of $\mathrm{LiCl}$. The latter procedure controlled for the ingestion-toxin contingency in the conditioning groups. Hereafter, controls will be called ingestion-toxin controls (ITC). Day 7 was the recovery day: all rats received $10 \mathrm{~min}$ access to distilled water at 8:30 a.m. and 3:30 p.m. On Days 8-15, at 8:30 a.m., half of the rats $(n=7)$ in each COND group received $10 \mathrm{~min}$ access to $5 \%$ ethanol, and half received distilled water. In each ITC group, 5 rats received access to $5 \%$ ethanol and 6 received distilled water. At 3:30 p.m. on Days 8-15, all rats had ad lib access to food and 10 min access to distilled water.

Days 16-23 were aversion test days, with one trial per day. Each trial provided $10 \mathrm{~min}$ access to a single bottle containing the alcoholic beverage CS. The measure of performance was the amount drunk, calculated to the nearest $100 \mathrm{mg}$ of the difference between pretest and posttest bottle weights.

Beverage class CSs and their content of congeners (milligrams per standard drink) were: for wine, Martini and Rossi dry vermouth (108.4 mg) and Gallo Burgundy (62.9 mg); for distilled spirits, Dewar's Scotch (48.6 mg) and Gordon's gin (6.8 mg) (see Franchina \& Dyer, 1986; Greizerstein, 1981). Each beverage was diluted to a $5 \%(v / v)$ ethanol concentration by mixing the original concentration with deionized distilled water. The $5 \%(\mathrm{v} / \mathrm{v})$ ethanol solution was presented during the period between aversion training and testing. I performed analyses of variance (ANOVAs) and $t$ tests on the data as appropriate $(\alpha=0.05)$.

\section{RESULTS}

To evaluate whether aversion conditioning to wine or distilled spirits CSs generalized to influence subsequent ethanol intake, I determined mean ethanol intakes in the
Table 1

Mean Intake (in grams) of Ethanol During Access Periods 1-3

\begin{tabular}{cccc}
\hline \multicolumn{4}{c}{ Periods } \\
\cline { 2 - 4 } CS & 1 & 2 & 3 \\
\hline \multicolumn{4}{c}{ Conditioned Group } \\
Wine-HI & 2.5 & 9.8 & 10.0 \\
Wine-LO & 1.8 & 7.4 & 11.3 \\
DiSp-HI & 1.6 & 7.0 & 11.5 \\
DiSp-LO & 2.3 & 6.7 & 10.2 \\
\multicolumn{4}{c}{ Ingestion-Toxin Control Group } \\
Wine-HI & 5.8 & 12.1 & 10.1 \\
Wine-LO & 8.9 & 13.3 & 11.8 \\
DiSp-HI & 4.0 & 9.9 & 8.8 \\
DiSp-LO & 5.0 & 9.6 & 10.7 \\
\hline
\end{tabular}

Note-HI and LO refer to high and low levels of congener in wine or distilled spirits (DiSp).

first three ethanol access periods (Periods 1-3) between training and testing (see Table 1). Ethanol intake was lower for COND groups than for ITC groups in Periods 1 and $2[F(1,40)=36.53, p<.001$, and $F(1,40)=11.19$, $p<.002$, respectively]. There were no reliable differences in ethanol intake across CS groups in the COND condition $(F<1)$. For the ITC condition, however, ethanol intake was lower for distilled spirits CS groups than for wine CS groups in each period $[F(1,16)=4.21$, $p<.06$, and $F(1,16)=4.38, p<.05$, respectively]. From Period 3 onward there were no reliable differences in ethanol intake between COND and ITC conditions or among CS groups. Considered over all access periods, ethanol intake was reliably below distilled water intake for all groups $(p<.001)$. Finding a reliable difference in ethanol intake between COND and ITC groups in Periods 1 and 2 suggests that aversion effects generalized from the beverage CSs to ethanol, irrespective of the congener contents of the beverage CSs.

Figure 1 shows mean intake (in grams) for each beverage $\mathrm{CS}$ in aversion testing. For each CS, intake by COND

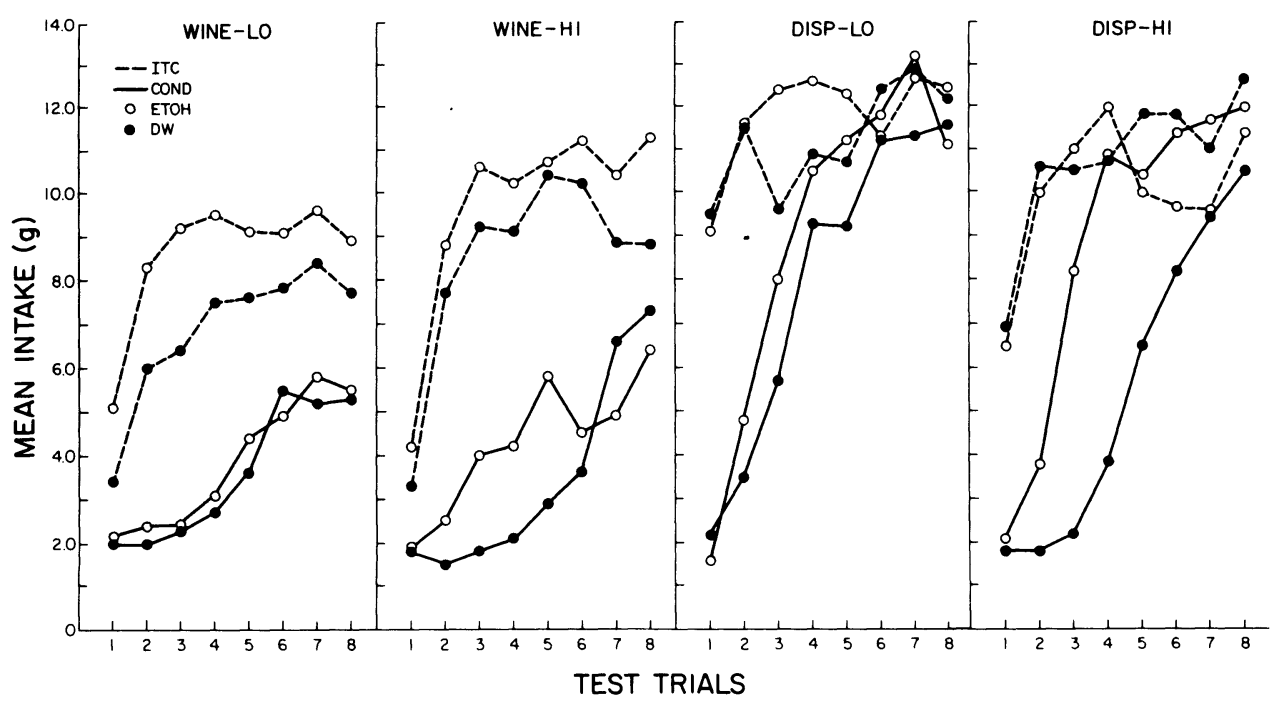

Figure 1. Mean intake (in grams) of wine and distilled spirits of low or high congener contents on Trials 1-8 for groups that received conditioning (COND) or ingestion-toxin control (ITC) procedures and then received access to ethanol (ETOH) or distilled water (DW) between aversion training and aversion test days. 
groups was below that of ITC groups on early trials. This difference persisted throughout testing for the wine CSs but dissipated for the distilled spirits CSs. For COND groups, access to ethanol between training and testing reduced the level of aversion (i.e., increased beverage intake) to a greater extent for wine and spirits CSs with high congener content than for those with low congener content (all relative to the effects of distilled water access). An ANOVA over all the results shown in Figure 1 yielded reliable effects for conditioning procedure $[F(1,84)=$ 121.95], beverage class $[F(1,84)=99.12]$, access fluid $[F(1,84)=6.65]$, beverage class $\times$ conditioning procedure $[F(1,84)=4.52]$, beverage class $\times$ congener level $[F(1,84)=7.40]$, beverage class $\times$ conditioning procedure $\times$ access fluid $[F(1,84)=5.51]$, and beverage class $x$ conditioning procedure $\times$ access fluid $\times$ congener level $\times$ test trials $[F(1,588)=46.21]$ (all $p$ s $<.05)$.

It is possible that the difference in aversion effects between wine and distilled spirits CSs and the effects of ethanol access could be attributable to the relative potability of the beverages and/or to the effects of ethanol access on that potability. To minimize these possibilities, I transformed the data of Figure 1 into percentage intake scores by dividing the intake of each subject in the COND group by the mean intake of its respective ITC group (see Franchina \& Dyer, 1986; Franchina et al., 1985). The lower the percentage score was, the greater the aversion effect.

Figure 2 presents mean percent intake for wine and distilled spirits CSs during test days. The results for wine intake on Trial 1 misleadingly suggest weak aversion effects. This finding may have reflected a floor effect in intake for COND groups or a suppression of intake, because of the novelty or potability of the wine CSs, for

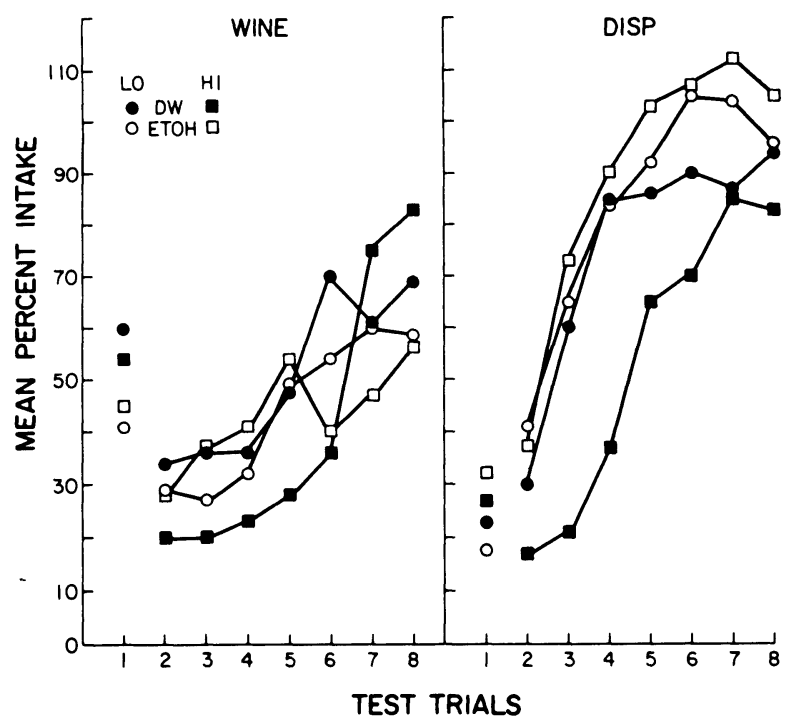

Figure 2. Mean percent intake for groups that were conditioned with wine or distilled spirits CSs of low (LO) or high (HI) congener contents and then were tested with the CS following interpolation of ethanol (ETOH) or distilled water (DW) between aversion training and test days.
ITC groups (see Figure 1, Trial 1; see also Franchina \& Dyer, 1986, for similar findings). Accordingly, because the data of Trial 1 may be artifactual, Figure 2 results should be considered from Trial 2 onward. Essentially, these data corroborated the conclusions derived from Figure 1. Aversion effects were generally greater and more persistent for wine than for distilled spirits CSs [for beverage class, $F(1,48)=23.04$, and for the beverage class $X$ trials interaction, $F(7,336)=16.5$; both $p s<.001$ ]. In each beverage class, groups that received distilled water access between training and testing (DW groups) showed stronger aversion effects to the beverage CS that was high in congener contents [congener level $\times$ trials for wine, $F(7,168)=3.62$, and for distilled spirits, $F(7,168)=7.50$; both $p s=.001]$. Conversely, relative to the results for DW groups, in each beverage class group, ethanol access reduced the aversion more to the CSs that were high in congeners [wine-high access fluid $\times$ test trials, $F(7,84)$ $=3.57, p<.001$; distilled-spirits-high access fluid, $F(1,12)=11.81, p<.001]$. There were no reliable effects of access fluid (ETOH vs. DW) for wine and distilled spirits CSs in the low-congener condition $(F \mathrm{~S}<1)$.

\section{DISCUSSION}

The present results for raw intake and percent intake corroborated previous findings from Franchina and Dyer (1986): aversion effects were generally greater for wine CSs than for distilled spirits CSs. For each of these beverage classes, aversion effects were greater with higher contents of congeners in the beverage CS (provided that only distilled water access occurred between aversion training and testing).

The most important finding of this experiment, however, was unexpected. For each beverage class CS, ethanol access between training and testing reduced the aversion more to the beverage with high congener content than to the beverage with low congener content. These results seem contrary to an associative analysis of compound conditioning (Rescorla \& Wagner, 1972). An associative analysis proposes that the conditionability of an elemental CS, such as ethanol, in a compound CS, such as Scotch, may depend upon the availability of other stimulus elements (e.g., congeners) for conditioning. Thus, ethanol should more likely be conditionable as an elemental CS in a beverage whose content of congeners is low (e.g., in gin) rather than high (e.g., in Scotch). If so, then ethanol access between training and testing should have extinguished the aversion to the conditioned ethanol stimulus more readily in beverages with low contents of congeners (e.g., Burgundy wine or gin) than in beverages with high congener contents (e.g., dry vermouth or Scotch). Reduction of the overall aversion to the beverage CS should follow pari passu. The present results show exactly the reverse effects.

It is possible that although ethanol and congeners are chemically separable by gas-liquid chromatography (Greizerstein, 1981), their stimulus characteristics that contribute taste and aroma to the alcoholic beverage may be less readily separable to the rat. Thus, the present results may reflect a degree of commonality between the stimuli occasioned by ethanol and those from congeners as they occurred in compound in the alcoholic beverages in this study. For example, taste and aroma characteristics in wine result primarily from esters, mainly ethyl acetate, and then from the higher alcohols (fusel oil), such as glycerol, butylene glycol, and acetyl methyl carbinol (Leake \& Silverman, 1971, p. 588). In distilled spirits, taste and aroma characteristics result mainly from the higher alcohols which include propyl, butyl, amyl, and their various isomers (Leake \& Silverman, p. 608).

Esters are less contributory to taste and smell in spirits. Furthermore in each beverage class, a beverage with a high content of congeners would contain more parts per million (ppm) of the various congeners, 
such as esters, the higher alcohols, aldehydes, methanol, and tannins. For example, Leake and Silverman (1971) reported that dry vermouth contained $388 \mathrm{ppm}$ of higher alcohols; a red wine, such as Burgundy, $298 \mathrm{ppm}$; Scotch, $808 \mathrm{ppm}$; and gin, 13 ppm. If the congeners (e.g., higher alcohols) in a beverage CS contribute taste and aroma characteristics that share stimulus characteristics with ethanol, then ethanol may not be a separable conditioning element in that beverage CS. Consequently, greater aversion conditioning may occur to a beverage CS whose content of congeners is high rather than low; however, if that beverage's congeners occasion stimuli similar to those from ethanol, the conditioned aversion may be susceptible to generalized extinction from subsequent access to ethanol, as in this study. Figure 2 shows that ethanol access reduced aversion effects to distilled spirits more than to wine, especially in the high-congener condition. This effect may have occurred because of a greater similarity of stimulus characteristics between ethanol and the higher alcohols in distilled spirits than between ethanol and esters in wine. Within a beverage class, such as distilled spirits, Scotch had a higher content of congeners than did gin and, thus, had more stimuli available for stronger aversion conditioning than did gin (see results for DW groups in both figures). If the congeners of Scotch (e.g., the higher alcohols) produced stimulus characteristics that were similar to those from ethanol, however, the conditioned aversion to Scotch may have been more susceptible to extinction from ethanol access between training and testing than was the conditioned aversion to gin.

\section{REFERENCES}

Franchina, J. J., \& DYer, A. B. (1986). Congener characteristics influence aversion conditioning to alcoholic beverages in rats. Behavior Research \& Therapy, 24, 299-306.

Franchina, J. J., Dyer, A. B., Gilley, D. W., Ness, J., \& DodD, M. (1985). Role of ethanol in conditioning aversion to alcoholic beverages in rats. Behavior Research \& Therapy, 23, 521-529.

GreizersteIN, H. B. (1981). Congener contents of alcoholic beverages. Journal of Studies of Alcohol, 42, 1030-1037.

LEAKE, C. D., \& Silverman, M. (1971). The chemistry of alcoholic beverages. In B. Kissin \& H. Begleiter (Eds.), The biology of alcoholism: Biochemistry (Vol. 1, pp. 575-612). New York: Plenum Press.

Rescorla, R. A., \& Wagner, A. R. (1972). A theory of Pavlovian conditioning: Variations in the associability of stimuli with reinforcement. In A. H. Black \& W. F. Prokasy (Eds.), Classical conditioning II: Current theory and research (pp. 64-99). New York: Academic Press.

ReVUSKY, S., \& TAUKULIS, H. K. (1975). Effects of alcohol and lithium habituation on the development of alcohol aversion through contingent lithium injection. Behavior Research \& Therapy, 13, 163-166.

SNELL, C. A. (1958). The congener content of alcoholic beverages. Quarterly Journal of Studies of Alcohol, 19, 69-71. 